\title{
From open radical hysterectomy to robot-assisted laparoscopic radical hysterectomy for early stage cervical cancer: aspects of a single institution learning curve
}

\author{
H. W. R. Schreuder • R. P. Zweemer • W. M. van Baal • \\ J. van de Lande $\cdot$ J. C. Dijkstra $\cdot$ R. H. M. Verheijen
}

Received: 18 January 2010 / Accepted: 12 February 2010/Published online: 13 April 2010

(C) The Author(s) 2010. This article is published with open access at Springerlink.com

\begin{abstract}
We analysed the introduction of the robotassisted laparoscopic radical hysterectomy in patients with early-stage cervical cancer with respect to patient benefits and surgeon-related aspects of a surgical learning curve. A retrospective review of the first 14 robot-assisted laparoscopic radical hysterectomies and the last 14 open radical hysterectomies in a similar clinical setting with the same surgical team was conducted. Patients were candidates for a laparoscopic sentinel node procedure, pelvic lymph node dissection and open radical hysterectomy (RH) before August 2006 and were candidates for a laparoscopic sentinel node procedure, pelvic lymph node dissection and robot-assisted laparoscopic radical hysterectomy (RALRH) after August 2006. Overall, blood loss in the open cases was significantly more compared with the robot cases. Median hospital stay after RALRH was 5 days less than after RH. The median theatre time in the learning period for
\end{abstract}

Information about relevant financial relationships outside the submitted work:

R.H.M. Verheijen: Financial relationships with any entities that have an interest related to the submitted work: Consultancy, Dues to be received, Proctorship for Intuitive Surgery

H. W. R. Schreuder $(\bowtie) \cdot$ R. P. Zweemer $\cdot$ R. H. M. Verheijen Division of Woman and Baby,

Department of Surgical and Oncological Gynaecology,

University Medical Centre Utrecht,

P.O. Box 85500, Room F05-126,

3508 GA, Utrecht, The Netherlands

e-mail: h.w.r.schreuder@umcutrecht.nl

R. H. M. Verheijen

e-mail: r.verheijen@umcutrecht.nl

R. P. Zweemer • W. M. van Baal · J. van de Lande •

J. C. Dijkstra $\cdot$ R. H. M. Verheijen

Department of Obstetrics and Gynaecology,

VU University Medical Centre,

Amsterdam, The Netherlands the robot procedure was reduced from $9 \mathrm{~h}$ to less that $4 \mathrm{~h}$ and compared well to the $3 \mathrm{~h}$ and $45 \mathrm{~min}$ for an open procedure. Three complications occurred in the open group and one in the robot group. RALRH is feasible and of benefit to the patient with early stage cervical cancer by a reduction of blood loss and reduced hospital stay. Introduction of this new technique requires a learning curve of less than 15 cases that will reduce the operating time to a level comparable to open surgery.

Keywords Robotic surgery $\cdot$ da Vinci $\cdot$ Radical hysterectomy · Learning curve

\section{Introduction}

For the surgical treatment of stage Ib1 cervical cancer, open radical hysterectomy with pelvic lymph node dissection has been the gold standard for over 100 years and has undergone little modification since it was first described by Wertheim [1] and later by Meigs [2]. Techniques for laparoscopic radical hysterectomy and lymph node dissection were developed in the early 1990s [3, 4] and considerable experience has been gained since with over 1,000 cases reported in literature [5].

After initial experience with robotic systems that are no longer available, the introduction of the da Vinci Surgical Robotic system (Intuitive Surgery, Mountain View, Ca, USA) and subsequent FDA approval for gynaecological use in 2005 [6], has made the laparoscopic approach to complex radical gynaecologic operations more feasible. The robotic system has an advantage over the traditional laparoscopic approach regarding improved articulation of instruments, stereoscopic vision, tremor reduction and motion downscaling [7]. These features suggest that a 
shorter learning curve may be achieved compared to conventional laparoscopy [8]. Robot-assisted laparoscopic surgery is used in gynaecology for benign hysterectomy, myomectomy, tubal reanastomoses, radical surgery, lymph node dissections and sacrocolpopexias [9, 10]. Robotassisted laparoscopic radical hysterectomy (RALRH) for cervical cancer has been recently introduced. A total of 313 cases in 11 reports have now been published [11-20]. These early reports describe operation time, blood loss, hospital stay, lymph node count and complications, but the aspect of the learning curve is not well described. One report describes the aspect of the learning curve more in detail [21]. In the present study, we compare 14 open radical hysterectomies (RH) with 14 RALRHs including sentinel node detection. We analysed patient-related aspects (blood loss, operating time, radicality of surgery (lymph node count), hospital stay and follow-up) and surgeonrelated aspects (operating time) of the learning curve of the surgical team in the transition from open to robot-assisted laparoscopic radical hysterectomy.

\section{Material and methods}

A total of 14 cases underwent an open RH between July 2004 and July 2006, and subsequently 14 patients were operated with the use of the da Vinci robot between August 2006 and January 2008 at the VU University Medical Centre in the Netherlands. All the operations were performed by the same surgical team. In one case from the Robot group, a sentinel lymph node showed metastatic disease, and the radical hysterectomy was abandoned. The patient received chemo-radiation therapy. This case was excluded from further analysis. One case in the Robot group consisted of a stage IIb endometrial cancer. All other cases where FIGO stage IBI cervical cancer.

In the Netherlands, we introduced the laparoscopic pelvic lymph node dissection (LPLND) with sentinel node (SN) detection for the surgical treatment of FIGO Ia2-IIa cervical cancers in 2000 [22]. When the SN contains a metastasis, the procedure is abandoned, and the patient subsequently receives chemo-radiotherapy. The aim of this approach is to reduce the number of patients undergoing radical hysterectomy followed by chemo-radiation as this leads to substantially more morbidity than either treatment alone, without obvious better survival [23, 24].

Our surgical team first obtained experience with the da Vinci robot system in a training facility; consequently, experience was broadened on pelvic lymphadenectomy and sentinel node procedures. Also, simple hysterectomies were performed with the system while continuing to perform radical hysterectomies by laparotomy. We subsequently started to perform robotic radical hysterectomies, using da
Vinci Robotic system with four robotic arms (Intuitive Surgery, Mountain View, Ca, USA) (Fig. 1), in August 2006. Before and after this date, the last open procedures and the first robotic procedures by the same team were compared with respect to number of lymph nodes retrieved (as a marker for radicality of surgery), theatre time, blood loss, hospital stay and complications.

Technically, the robot-assisted procedure starts with the injection of Tc99-nanocol the day prior to surgery and a scintigram is made to detect and localise the SNs. On the day of surgery, immediately prior to the procedure $2-\mathrm{ml}$ patent blue (Patent Blue V, 2.5\% solution, Laboratoire Guerbet, Roissy, France) is injected in the cervix around the tumour. A three-way urinary catheter is introduced. A McCartney tube $(4.5 \mathrm{~cm})$ [25] is inserted in the vagina and fixed to the cervix, by means of a suture through the cervix, which is attached to the end of the tube. The abdomen is insufflated through a Verres needle just above the umbilicus where an $11-\mathrm{mm}$ camera port is introduced. Two $8-\mathrm{mm}$ robotic troicarts are introduced at the level of and $10 \mathrm{~cm}$ laterally to the supra-umbilical incision. The third 8-mm robotic port is introduced on the right lower lateral abdomen. A 5th 11-mm port is introduced in the left upper quadrant for the assistant. The patient is then placed in extreme Trendelenburg position; and at this point, the robot system is attached. The procedure then continues with opening of the retroperitoneum lateral to the external iliac artery and all spaces paravesical and pararectal as well as the obturator fossa are opened in search for the SN, both visually and by use of a laparoscopic radiosensitive probe (Europrobe, Strassbourg, France) (Fig. 2). When the SNs are

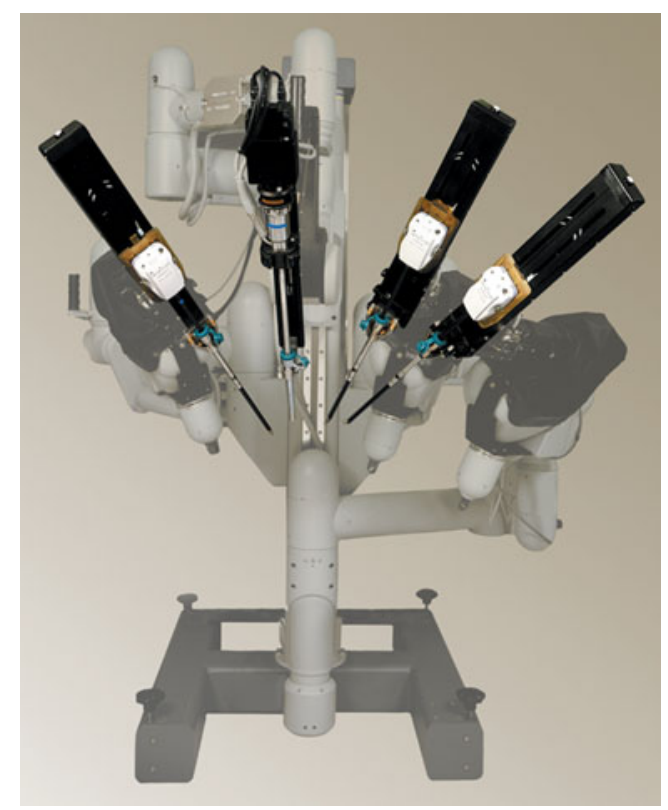

Fig. 1 Four arm da Vinci robot (Intuitive Surgery, Mountain View, $\mathrm{Ca}, \mathrm{USA}$ ) 


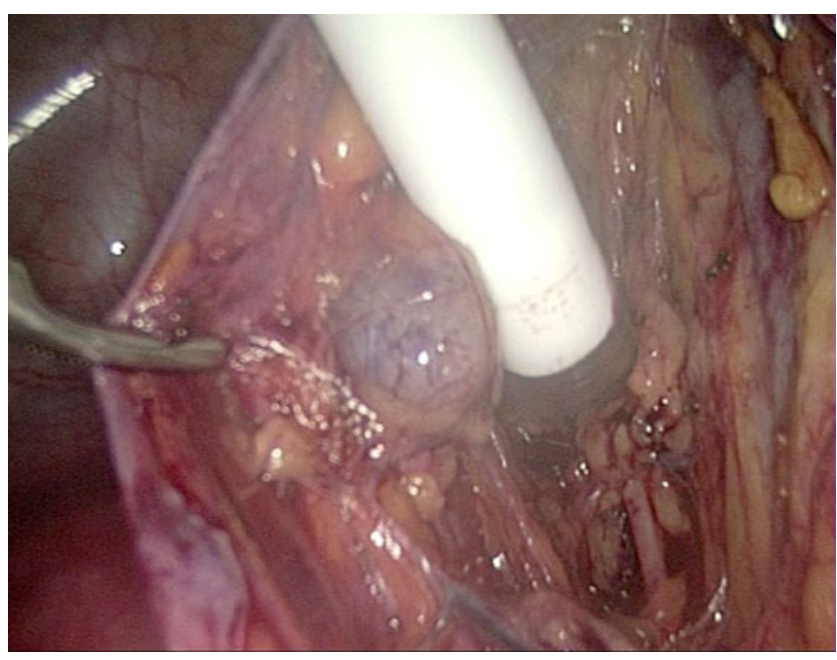

Fig. 2 Sentinal node detection after injection of patent blue and with a laparoscopic radiosensitive probe

identified and selectively removed they are sent for frozen section. The LPLND is then systematically completed. If the $\mathrm{SN}$ contains no metastasis, the radical hysterectomy is started by dissection of the ovarian vessels and uterine artery by bipolar coagulation and selective coagulation and cutting. The ureters are mobilised to the level of the ureteric tunnel. The round ligaments are then coagulated and cut and the bladder dissected downward. The fourth robotic arm is used to manipulate the uterus contra laterally. The posterior peritoneum is then incised, and the rectum deflected. The sacrouterine ligaments are cut. The parametria are then cut. The vaginal cuff is identified on the McCartney tube and cut by monopolar coagulation. The specimen can now be removed vaginally with the McCartney tube, and the vaginal vault was sutured. All radical hysterectomies were performed at the Rutledge type III level [26] both for the open and Robotic procedure. Statistical analysis was done using the statistical software package SPSS 15.0 (SPSS Inc, Chicago, IL). The outcome for robot-assisted and open groups were compared using the Chi-square test and Fisher extract test for categorical variables and two sample student $t$ test for continuous variables. A $P$ value of 0.05 was considered significantly.

\section{Results}

The median age in the robot group is 43 years compared to 46 years in the open group. The distribution of histology was similar in both groups, squamous cell carcinoma's predominated. In the open group high grade histology predominated whereas in the robot group most cases were graded as moderately differentiated (Table 1).

The median number of lymph nodes removed was slightly higher in the robot group 29 versus 26 in the open group (not statistically significant) (Table 2). Blood loss was calculated by the difference in the total amount of suctioned and irrigated fluids. Median blood loss in the open group was 2,000 $\mathrm{ml}$ (range, 1,000-4,600 ml). For the robot group, median blood loss was $300 \mathrm{ml}$ (range, 50$1,000 \mathrm{ml}$ ). In both groups, blood loss for SN+LPLND and radical hysterectomy was combined. Hospital stay for the radical hysterectomy in the open group was at a median of 9 days (range, 7-16 days). In the robot group, the median hospital stay was 4 days (range, 3-14 days). Three complications occurred in the open group. One cystotomy lesion was managed conservatively. One temporary ureteric obstruction occurred post-operatively, and the hydronefrosis was decompressed by percutaneous drainage. After removal of this drain, the patient recovered well. A vault abscess was drained in a third patient. In the robot group, one complication occurred: an accessory ureter was cut requiring percutaneous drainage and re-anastomosis in a second procedure. Theatre times are represented in real theatre time spent and are calculated from the start of anaesthetic preparations. Theatre time ends when the patient leaves the operating table. For the open group, the median theatre time for the radical hysterectomy was

Table 1 Patient characteristics, histology and stage

\begin{tabular}{llll}
\hline & Robot group & & Open group \\
\hline$N$ & 13 & & 14 \\
Age & Median (range) & $43(31-78)$ & $46(32-68)$ \\
Histology & Squamous (\%) & $10(77)$ & $11(79)$ \\
& Adeno (\%) & $2(15)$ & $3(21)$ \\
& Endometrioid (\%) & $1(8)$ & $0(0)$ \\
Grade & 1 & 0 & 0 \\
& 2 & 9 & 6 \\
\multirow{4}{*}{ Stage (FIGO) } & Ib1 & 4 & 8 \\
& 3 & 11 & 12 \\
& Other & $($ One endometrial cancer stage IIB, one stage Ib2 & $($ One stage Ib2) \\
\hline
\end{tabular}


Table 2 Comparison of the theatre time, lymph nodes removed, blood loss, hospital stay, complications and recurrences for radical hysterectomy patients

\begin{tabular}{llll}
\hline & Robot group $(n=13)$ & Open group $(n=14)$ & $P$ value \\
\hline $\begin{array}{l}\text { Theatre time (min) } \\
\text { Median (range) }\end{array}$ & $434(264-610)$ & $225(170-330)$ & $<0.001$ \\
$\begin{array}{l}\text { LN removed ( } n) \\
\text { Median (range) }\end{array}$ & $29(19-76)$ & $26(10-41)$ & 0.064 \\
$\begin{array}{l}\text { Blood loss (ml) } \\
\text { Median (range) }\end{array}$ & $300(50-1,000)$ & $2,000(1,000-4,600)$ & $<0.001$ \\
$\begin{array}{l}\text { Hospital stay (days) } \\
\text { Median (range) } \\
\begin{array}{l}\text { Complications }(n) \\
\text { Number }\end{array}\end{array}$ & $4(2-14)$ & $9(7-16)$ & $<0.001$ \\
$\begin{array}{l}\text { Follow-up (months) } \\
\text { Median (range) } \\
\text { Recurrences }(n)\end{array}$ & 1 & 3 & - \\
\begin{tabular}{l} 
Number \\
\hline
\end{tabular} & $26(17-32)$ & $42(31-54)$ & $<0.001$ \\
\end{tabular}

$3 \mathrm{~h}: 45$ min (range, 2:50-5:30). For the robot group, this was $7 \mathrm{~h}: 23 \mathrm{~min}$ (range, 4:24-10:10) and includes the $\mathrm{SN}+\mathrm{LPLND}$ in 10 of 13 cases. In the remaining three cases, the SN+LPLND was performed in a separate surgical session, and the console time of the SN+LPLND was added to the total theatre time of the radical hysterectomy. The theatre times are decreasing dramatically over time in the robot group, whereas theatre time in the open group has obviously remained stable (Fig. 3).

In a median follow-up of 42 months (range, 31-54), for the open group, one pelvic sidewall recurrence occurred 7 months after the radical hysterectomy. The robot group has a mean follow-up of 26 months (range, 17-32) in which two recurrences occurred. One pelvic sidewall recurrence and a port metastasis occurred 12 months after primary surgery. This was treated with excision of the port metastasis and chemo-radiation on the pelvis. The second recurrence occurred 17 months after primary surgery and was located in the rectum, a posterior exenteration was performed. All but one patient received single modality treatment. In one patient from the open group, postoperative radiotherapy was required due to a narrow vaginal margin.

\section{Comment}

In this study, we could confirm the findings of previous authors describing the RALRH for the treatment of cervical cancer $[11,13-18,20]$. They also conclude that this technique is feasible and could be of benefit to the patient regarding reduction of blood loss and reduction of hospital stay. We found a significant reduction of blood loss, and hospitalisation was reduced by 5 days in the robotic group indicating significant quicker recovery compared to open surgery. One aspect of quality of surgery is the number of pelvic lymph nodes removed. In this study, lymph node counts were higher in the robotic group, although this was not statistically significant. Most other authors could not find a statistically significant difference in lymph node counts compared with open surgery, but there seems to be a trend towards a higher lymph node retrieval in patients operated with robot assistance [14, 15, 17]. An explanation could be that the studies were too small to show a statistically significant difference. Recent larger reports concerning the RALRH confirm this trend and found a statistically significant higher lymph node count in the robotic group [11, 12]. It is likely that this may be attributed to instrument articulation, $3 \mathrm{D}$ view and motion downscaling, allowing a very selective dissection in robot-assisted surgery. In our study, the complication rate was lower in the robot-assisted group; this is consistent with the data described by other authors [11, 12, $14,15]$. There where twice the number of recurrences in the robotic group, and the follow-up was shorter in the robotic group (Table. 2). However, numbers are too small to draw

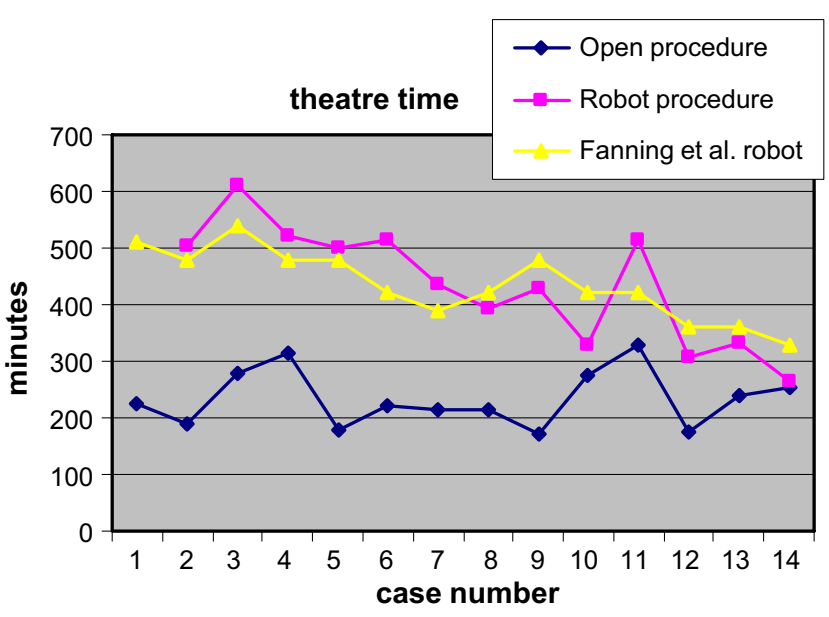

Fig. 3 Theatre time for the Radical Hysterectomy (open procedure and robot procedure) also plotted the learning curve from Fanning et al. [21] 
statistical conclusions for recurrences and complication rates. In addition, we showed that theatre time may rapidly be reduced to the level of an open radical hysterectomy. A learning curve of 14 cases reduced the team's total theatre time by $48 \%$. Our learning curve is comparable with the only other published detailed learning curve for the RALRH described by Fanning et al. [21]. We plotted their learning curve of the first 14 robotic cases together with our learning curve (Fig. 3). Their reduction in theatre time is $35 \%$ after 14 cases and $59 \%$ after 20 cases. This is suggesting a relatively short learning curve for robotic procedures. Person et al. reported their skin-to-skin time for the RALRH (with or without sentinel node) and found also a rapid decrease of surgery time [19]. The learning curve for the robot hysterectomy with pelvic and para-aortic lymphadenectomy for endometrial cancer is described in detail by Seamon et al. They conclude the learning curve for this procedure seems to be approximately 20 cases; however, efficiency continues to improve after the first 20 cases [27].

Our team gradually introduced the use of the robot and had experience in laparoscopic oncological surgery and open radical surgery for many years. When a more rapid transition to robotic surgery is chosen, the learning curve may be longer. In our study, we purposely measured real theatre time as it is our experience that a significant amount of time may be lost in preparing and positioning of the patient as well as positioning and introducing the robotic system. This is also stated by Seamon et al. who looked in detail at theatre time, skin-to-skin time and console times for the robotic hysterectomy with lymphadenectomy for endometrial cancer. In their study console, time is approximately half of the total theatre time [27]. Measuring pure console time, as is done in many other studies on robotic surgery, does not reflect the effort of the whole team's learning curve and may therefore not represent a true comparison with open surgery. In our study, the theatre time may be longer than in other centres. This is mainly due to the SN technique where the anatomical spaces are all opened before starting the lymph node in search of the SN. This procedure increases theatre time with approximately $1 \mathrm{~h}[28]$.

A dedicated surgical and anaesthesiological team tremendously increases efficiency and reduces the time spent in this phase of the procedure. Anaesthesiological aspects of robotic surgery should be well appreciated before embarking on robotic surgery [29, 30]. Interestingly, anaesthesiological complications did not occur despite prolonged surgical times with the patient in extreme Trendelenburg position.

There is still a lack of literature about the learning curve for robot-assisted gynaecological procedures. In urology, the aspect of the learning curve for radical prostatectomies is well described, and compared to conventional laparoscopy, the learning curve for robotic surgery is significantly shorter $[31,32]$. Recently, the learning curve of the robotassisted sacrocolpopexy was described, a reduction of operative time of $25 \%$ after ten cases was found, again suggesting a relatively short learning curve [33]. Two other studies have specifically looked at learning curves in benign gynaecology. Lenihan et al. performed 113 robotassisted procedures (mainly hysterectomies, but also myomectomy, sacrocolpopexy and oophorectomy). With the use of a dedicated team, they were able to set up the robot for surgery in $45 \mathrm{~min}$ after 20 cases and in 35 min within 50 cases. Robot console times and total operative times were consolidated after approximately 50 cases at about $50 \mathrm{~min}$ for console time and $90 \mathrm{~min}$ for total operative time [34]. Forty cases of benign gynaecologic procedures by a single surgeon are described by Pitter et al. After 20 cases, a statistical improvement in operative time is reached [35]. Both studies do not differentiate between the procedures performed.

In conclusion, robotic-assisted surgery is rapidly growing and has a high potential. Our data suggest a relatively short learning curve with quick improvement of patient and surgeon-related parameters. This is a great advantage in the implementation in daily practice.

Conflict of interest No payment or support in kind for any aspect of the submitted work (including but not limited to grants, data monitoring board, study design, manuscript preparation, statistical analysis, etc) was received

RHM Verheijen is a proctor for radical robot-assisted surgery, sponsored by Intuitive Surgery. For all other authors there is no conflict of interest.

Open Access This article is distributed under the terms of the Creative Commons Attribution Noncommercial License which permits any noncommercial use, distribution, and reproduction in any medium, provided the original author(s) and source are credited.

\section{References}

1. Wertheim E (1912) The extended abdominal operation for carcinoma uteri. Am J Obstet Gynecol 169-232

2. Meigs J (1945) The Wertheim operation for carcinoma of the cervix. Am J Obstet Gynecol 40:542-543

3. Canis M, Mage G, Wattiez A, Pouly JL, Manhes H, Bruhat MA (1990) Does endoscopic surgery have a role in radical surgery of cancer of the cervix uteri? J Gynecol Obstet Biol Reprod (Paris) 19(7):921

4. Nezhat CR, Burrell MO, Nezhat FR, Benigno BB, Welander CE (1992) Laparoscopic radical hysterectomy with paraaortic and pelvic node dissection. Am J Obstet Gynecol 166(3):864-865

5. Zakashansky K, Bradley WH, Nezhat FR (2008) New techniques in radical hysterectomy. Curr Opin Obstet Gynecol 20(1):14-19 
6. Food and Drug Association. www fda gov/fdac/features/2005/ 405_computer html. Accessed 2009 May 20

7. Camarillo DB, Krummel TM, Salisbury JK Jr (2004) Robotic technology in surgery: past, present, and future. Am J Surg 188 (4A Suppl):2S-15S

8. Blavier A, Gaudissart Q, Cadiere GB, Nyssen AS (2007) Comparison of learning curves and skill transfer between classical and robotic laparoscopy according to the viewing conditions: implications for training. Am J Surg 194(1):115-121

9. Schreuder HW, Verheijen RH (2009) Robotic surgery. BJOG 116 (2):198-213

10. Visco AG, Advincula AP (2008) Robotic gynecologic surgery. Obstet Gynecol 112(6):1369-1384

11. Boggess JF, Gehrig PA, Cantrell L, Shafer A, Ridgway M, Skinner EN et al (2008) A case-control study of robot-assisted type III radical hysterectomy with pelvic lymph node dissection compared with open radical hysterectomy. Am J Obstet Gynecol 199(4):357.e1-357.e7

12. Estape R, Lambrou N, Diaz R, Estape E, Dunkin N, Rivera A (2009) A case matched analysis of robotic radical hysterectomy with lymphadenectomy compared with laparoscopy and laparotomy. Gynecol Oncol 113(3):357-361

13. Kim YT, Kim SW, Hyung WJ, Lee SJ, Nam EJ, Lee WJ (2008) Robotic radical hysterectomy with pelvic lymphadenectomy for cervical carcinoma: a pilot study. Gynecol Oncol 108(2):312-316

14. Ko EM, Muto MG, Berkowitz RS, Feltmate CM (2008) Robotic versus open radical hysterectomy: a comparative study at a single institution. Gynecol Oncol 111(3):425-430

15. Lowe MP, Hoekstra AV, Jairam-Thodla A, Singh DK, Buttin BM, Lurain JR et al (2009) A comparison of robot-assisted and traditional radical hysterectomy for early-stage cervical cancer. J Robotic Surg 3(1):19-23

16. Lowe MP, Chamberlain DH, Kamelle SA, Johnson PR, Tillmanns TD (2009) A multi-institutional experience with robotic-assisted radical hysterectomy for early stage cervical cancer. Gynecol Oncol 113(2):191-194

17. Magrina JF, Kho RM, Weaver AL, Montero RP, Magtibay PM (2008) Robotic radical hysterectomy: comparison with laparoscopy and laparotomy. Gynecol Oncol 109(1):86-91

18. Nezhat FR, Datta MS, Liu C, Chuang L, Zakashansky K (2008) Robotic radical hysterectomy versus total laparoscopic radical hysterectomy with pelvic lymphadenectomy for treatment of early cervical cancer. JSLS 12(3):227-237

19. Persson J, Reynisson P, Borgfeldt C, Kannisto P, Lindahl B, Bossmar T (2009) Robot assisted laparoscopic radical hysterectomy and pelvic lymphadenectomy with short and long term morbidity data. Gynecol Oncol 113(2):185-190

20. Sert B, Abeler V (2007) Robotic radical hysterectomy in earlystage cervical carcinoma patients, comparing results with total laparoscopic radical hysterectomy cases. The future is now? Int $\mathrm{J}$ Med Robot 3(3):224-228

21. Fanning J, Fenton B, Purohit M (2008) Robot radical hysterectomy. Am J Obstet Gynecol 198:649.e1-649.e4

22. Verheijen RH, Pijpers R, van Diest PJ, Burger CW, Buist MR, Kenemans P (2000) Sentinel node detection in cervical cancer. Obstet Gynecol 96(1):135-138

23. Landoni F, Maneo A, Colombo A, Placa F, Milani R, Perego P et al (1997) Randomised study of radical surgery versus radiotherapy for stage Ib-IIa cervical cancer. Lancet 350(9077):535-540

24. Richard SD, Krivak TC, Castleberry A, Beriwal S, Kelley JL III, Edwards RP et al (2008) Survival for stage IB cervical cancer with positive lymph node involvement: a comparison of completed vs. abandoned radical hysterectomy. Gynecol Oncol 109(1):43-48

25. McCartney AJ, Obermair A (2004) Total laparoscopic hysterectomy with a transvaginal tube. J Am Assoc Gynecol Laparosc 11 (1):79-82

26. Piver MS, Rutledge F, Smith JP (1974) Five classes of extended hysterectomy for women with cervical cancer. Obstet Gynecol 44 (2):265-272

27. Seamon LG, Cohn DE, Richardson DL, Valmadre S, Carlson MJ, Phillips GS et al (2008) Robotic hysterectomy and pelvic-aortic lymphadenectomy for endometrial cancer. Obstet Gynecol 112 (6): 1207-1213

28. Buist MR, Pijpers RJ, van Lingen A, van Diest PJ, Dijkstra J, Kenemans P et al (2003) Laparoscopic detection of sentinel lymph nodes followed by lymph node dissection in patients with early stage cervical cancer. Gynecol Oncol 90(2):290-296

29. Baltayian S (2008) A brief review: anesthesia for robotic prostatectomy. J Robotic Surg 2(2):59-61

30. Danic MJ, Chow M, Alexander G, Bhandari A, Menon M, Brown M (2008) Anesthesia considerations for robotic-assisted laparoscopic prostatectomy: a review of 1, 500 cases. J Robotic Surg 1:119-123

31. Artibani W, Fracalanza S, Cavalleri S, Iafrate M, Aragona M, Novara $G$ et al (2008) Learning curve and preliminary experience with da Vinci-assisted laparoscopic radical prostatectomy. Urol Int 80(3):237-244

32. Ficarra V, Cavalleri S, Novara G, Aragona M, Artibani W (2007) Evidence from robot-assisted laparoscopic radical prostatectomy: a systematic review. Eur Urol 51(1):45-55

33. Akl MN, Long JB, Giles DL, Cornella JL, Pettit PD, Chen AH et al (2009) Robotic-assisted sacrocolpopexy: technique and learning curve. Surg Endosc 23(10):2390-2394

34. Lenihan JP Jr, Kovanda C, Seshadri-Kreaden U (2008) What is the learning curve for robotic assisted gynecologic surgery? J Minim Invasive Gynecol 15(5):589-594

35. Pitter MC, Anderson P, Blissett A, Pemberton N (2008) Roboticassisted gynaecological surgery-establishing training criteria; minimizing operative time and blood loss. Int J Med Robot 4(2):114-120 